\title{
Influence of Cobalt lons on Collagen Gel Formation and Their Interaction with Osteoblasts
}

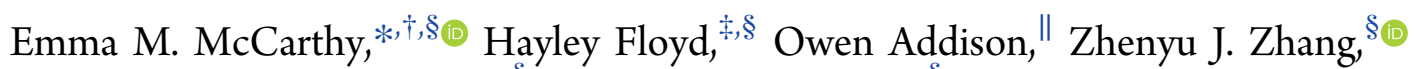 \\ Pola Goldberg Oppenheimer, ${ }^{\S}$ and Liam M. Grover*, ${ }^{*}$
} ${ }^{\dagger}$ Physical Sciences for Health, School of Chemistry, ${ }^{\ddagger}$ Physical Sciences of Imaging in the Biomedical Sciences, School of Chemistry,
${ }^{\S}$ Department of BioChemical Engineering, School of Chemical Engineering, and "School of Dentistry, University of Birmingham,
Edgbaston, B15 2TT Birmingham, U.K.

\section{Supporting Information}

ABSTRACT: Metals on metal implants have long been used in arthroplasties because of their robustness and low dislocation rate. Several relatively low-corrosion metals have been used in arthroplasty, including $316 \mathrm{~L}$ stainless steel, titanium, and cobalt-chromium-molybdenum alloy. Debris from these implants, however, has been found to cause inflammatory responses leading to unexpected failure rates approaching $10 \% 7$ years surgery. Safety assessment of these materials traditionally relies on the use of simple twodimensional assays, where cells are grown on the surface of the material over a relatively short time frame. It is now wellknown that the composition and stiffness of the extracellular matrix (ECM) have a critical effect on cell function. In this work, we have evaluated how cobalt ions influence the assembly of type I collagen, the principle component of the ECM in bone. We found that cobalt had a significant effect on collagen matrix formation, and its presence results in local variations in collagen density. This increase in heterogeneity causes an increase in localized mechanical properties but a decrease in the bulk stiffness of the material. Moreover, when collagen matrices contained cobalt ions, there was a significant change in how the cells interacted with the collagen matrix. Fluorescence images and biological assays showed a decrease in cell proliferation and viability with an increase in cobalt concentration. We present evidence that the cobalt ion complex interacts with the hydroxyl group present in the carboxylic terminal of the collagen fibril, preventing crucial stabilizing bonds within collagen formation. This demonstrates that the currently accepted toxicity assays are poor predictors of the longer-term biological performance of a material.

\section{INTRODUCTION}

Cobalt-chromium-molybdenum alloys are one of the most common alloys used for both metal-on-metal (MOM) arthroplasties and implant resurfacing because of their high level of robustness and low risk of dislocation. ${ }^{1-3}$ MOM implants have been found to have lower wear rates than metalon-polyethylene implants and generate smaller wear particles; ${ }^{4,5}$ however, there is a 500 -fold increase in the generation rate of these small particles. ${ }^{4-6}$ Metallic debris from MOM implants has been shown to initiate inflammatory responses which can lead to issues such as implant loosening and bone resorption. $^{7-10}$ In 2013, the Australian Orthopaedic Association National Joint Replacement Registry found unexpected failure rates approaching $10 \%$ after 7 years for MOM hip replacements alone. ${ }^{1}$ Furthermore, MOM implants are 1.5 times more likely to fail 2 years post primary surgery in comparison with metal-on-polyethylene implants. ${ }^{11}$ These failures were discovered because of the unexpected pain in patients, even in those with well-positioned implants, which suggested an adverse biological reaction to implant material derivatives rather than a biomechanical failure. ${ }^{12}$ Damage as a consequence of the release of particles and ions from the implant surface has been shown to affect both the bone and the surrounding soft tissue. ${ }^{13}$

In addition to the production of particulate debris due to wear, MOM implants are also susceptible to corrosion processes in vivo that lead to the generation of small particles (including nanoscale) and the release of implant metal ions. Tribocorrosion occurs at the surface of articulating components of MOM implants as a consequence of the combination of mechanical wear and localized corrosion. ${ }^{14}$ The unchallenged implant surface is protected from dissolution because of the presence of a passive surface oxide layer that limits corrosion. Disruption of this passive surface layer (due to mechanical damage) results in the exposure of the underlying metal, dissolution (anodic reaction), and the formation of

Received: May 18, 2018

Accepted: July 18, 2018

Published: August 30, 2018 
metal cations. The reaction produces electron flow from the corroding site to the metal surface (cathode), which is passive. Hydrolysis of released metal ions leads to a local acidification, which can subsequently allow free ions to easily migrate away from the original surface. ${ }^{15,16}$ Cobalt-chromium-molybdenum alloys are protected by a passive oxide layer, which is $1-4$ $\mathrm{nm}$ thick and primarily comprised chromium and cobalt oxides. The repetitive mechanical movement associated with load-bearing arthoplasties results in the abrasion of this thin oxide layer. Alongside the generation of particulate debris, the ionic cobalt and chromium released either remains in the solution or precipitates within the extracellular tissue space. As cobalt is more soluble than chromium, it is more likely to remain in the ionic form and interact with the extracellular matrix (ECM). ${ }^{14}$

Most investigations into how cobalt ions affect the surrounding tissue have focused on two-dimensional cell cultures, mimicking the osteolytic inflammatory response, driven by a direct interaction between macrophages and cobalt debris. ${ }^{17-20}$ In particular, cobalt ions have been found to interact with DNA and nuclear proteins, ultimately causing cell death. ${ }^{21}$ More specifically, cobalt ions are able to cross the cytoplasmic membrane, accumulating in the cell nucleus and subsequently the surrounding structures. ${ }^{21-23}$ It has also been found that cobalt ions cause apoptosis in macrophages after 24 $\mathrm{h}$ and necrosis after $48 \mathrm{~h}^{24,25}$ Furthermore, cobalt ions exacerbate inflammation by increasing the amount of proinflammatory cytokines that are released from macrophages, such as tumor necrosis factor-a, IL-1b, and Il6. ${ }^{19-21,26,27}$ In addition to this, two-dimensional cell cultures looking at the direct effect of cobalt ions on osteoblast/ mesenchymal stem cells have also been investigated. Studies showed how addition of cobalt ions into the cell media caused a decrease in the proliferation and function of osteoblasts and the differentiation of mesenchymal stem cells to osteoblasts. ${ }^{28-31}$ In addition to being a degradation product from metallic prostheses, cobalt has been used to stimulate the HIF 1a pathway and angiogenesis. Glasses loaded with up to $1 \mathrm{wt} \%$ have proven to be nontoxic with a maximal dose of $5 \mathrm{wt} \%$ before toxicity occurs. At this point, there are no reports on how much concentration may influence the ECM or ECM interactions. $^{32-34}$ However, it is well-known that cell interactions are driven by small changes in the threedimensional ECM, and therefore, two-dimensional studies are not sufficient. ${ }^{35}$

To date, studies in this area have investigated only the cellular effect of metal debris. However, it is the ECM that directly interacts with both implant and the surrounding tissue. The ECM mostly comprises collagen type I and provides not only structural support to cells and surrounding connective tissue but also plays a role within the differentiation of surrounding cells. ${ }^{35-40}$ Collagen type I chains comprise a triple amino acid repeating sequence, Gly-X-Y, where glycine residues occupy every third position, and the $\mathrm{X}$ and $\mathrm{Y}$ residues are usually proline and hydroxyproline, respectively. Three collagen chains bind together via hydrogen bonding to form a triple helical structure, typically spanning $300 \mathrm{~nm}$ in length. ${ }^{41}$ After both intra- and extracellular modification, the triple helix forms a collagen molecule. ${ }^{42}$ As collagen matrix formation is hierarchical, multiple stages are required prior to collagen fiber formation. Five-stranded microfibrils super-twist and quasihexagonally stack, via covalent and hydrogen bonding, to form a collagen fibril. Because of the parallel staggering formed from the super-twist, a regular overlapping pattern, referred to as $d$ spacing, can be observed. This typically measures approximately $67 \mathrm{~nm}^{43-45}$ Fibrils can range from 50 to a few hundred nanometers in thickness and aggregate, via covalent bonding, to form fibers. ${ }^{41}$ These fibers are cord-shaped and typically range within $1-20 \mu \mathrm{m}$ in diameter. ${ }^{43}$ Collagen fibers are the main constituents of the ECM and provide structural support to surrounding cells. As metallic ions produced from corrosion of MOM implants are so small, they can interact with the collagen fiber at much earlier stages of development. This can hinder the hierarchical process of collagen formation and may potentially alter both mechanical and structural properties of the matrix. Any changes to the structure of the ECM will drastically affect the way in which cells attach and thereby affect the tissue function. This study investigates how the presence of cobalt(II) ions affects collagen matrix formation and cell interactions to better understand unexpected MOM failures. Osteoblast cells were used to understand the effects of changes to the ECM without the influence of inflammatory changes. A maximum concentration of $200 \mathrm{ppm}$ was used as this is the highest recorded cobalt concentration at the injury site. ${ }^{46}$ This study highlights the importance of understanding the long-term biocompatibility of materials regarding not only cell response but also changes to the ECM.

\section{RESULTS}

The effect of cobalt ions on the fibrillogenesis of collagen formation was analyzed using UV-vis spectrophotometry. Turbidity measurements, as shown in Figure 1A, suggest that the cobalt ion concentration has a detrimental effect on the kinetics of collagen fibril assembly. It takes much longer for the fibrils to fully form with the addition of cobalt as shown by the extension of the growth phase. This is further shown by comparing the growth phase ratios with respect to $0 \mathrm{ppm}$, as shown in Figure 1B. Turbidity results suggest that even at low concentrations, the cobalt ions interact with the collagen fibrils. To determine the loss of cobalt ions from collagen hydrogels, a leaching assay was performed, as shown in Figure 1C. The $\mathrm{CoCol}$ gel with a cobalt concentration of $200 \mathrm{ppm}$ released only $8 \mathrm{ppm}$ of cobalt ions into the surrounding media after 6 days. Given the small size of the cobalt ions, ${ }^{4}$ it is unlikely that they are trapped sterically in the hydrogel matrix. This suggests that the ions are binding strongly within the hydrogel, preventing their diffusion into the media.

Atomic force microscopy (AFM) was performed to fully understand the effect of cobalt ions on the formation of collagen matrices. The microstructure of collagen fibrils doped with cobalt is shown in Figure 2. These images show that there are areas of both high- and low-density collagen fibrils in samples containing cobalt, as demonstrated in Figure 2A,B. These observations were also confirmed by reflectance microscopy images, shown in Figure $2 \mathrm{C}$, as there is more collagen agglomeration with the addition of cobalt. This indicates that the cobalt ions alter the localized structure of the collagen matrix.

Force spectroscopy measurements on the CoCol gels were performed to determine the effect of cobalt addition on the adhesiveness of the samples. Because there is no alteration to the chemical nature of the collagen gel, nanomechanical properties could effectively reflect the localized arrangement of collagen fibrils. Large adhesion forces measured by the AFM tip are due to an increased contact area as a result of a readily 
A
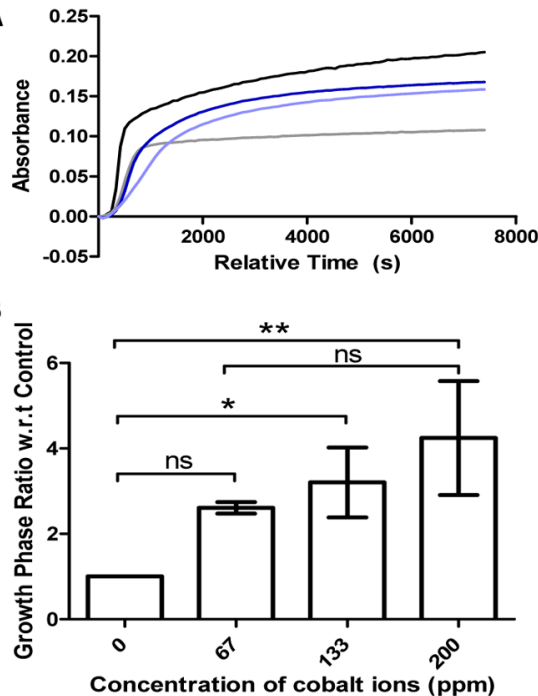

C

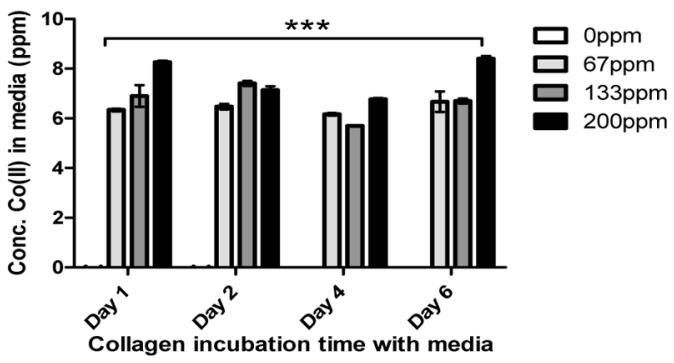

Figure 1. (A) Turbidity of cobalt-doped collagen at cobalt concentrations of $0,67,133$, and $200 \mathrm{ppm}$. (B) Ratio of the growth phases at each cobalt concentration with respect to $0 \mathrm{ppm}$. (C) Leaching of cobalt ions from collagen hydrogels at varying time points. The initial cobalt concentrations were $0,67,133$, and 200 ppm.

deformed collagen network and vice versa. Samples containing 0,67 , and $133 \mathrm{ppm}$ of cobalt appear to have both stiff and soft fibrils. However, adhesion forces of the $200 \mathrm{ppm}$ sample show that the fibrils are only soft, as shown in Figure 3. This result indicates that increased concentrations of cobalt ions could possibly block the important cross-linking binding sites for collagen and hence result in a porous matrix with enhanced local adhesiveness and reduced stiffness.

To analyze the dispersion of cobalt throughout the bulk matrix, X-ray fluorescence (XRF) measurements of CoCol gels were obtained, indicating the presence of cobalt ions, as depicted in Figure 4. It was shown that there were regions of localized cobalt, instead of an even dispersion, with an average 3 -fold increase in cobalt concentration at areas of high intensity. This suggests that the increase in the heterogeneity of collagen fibrils within CoCol gels may be due to the uneven distribution of cobalt ions.

The effects of the heterogeneous dispersion of cobalt on the bulk material properties of collagen hydrogels were determined using oscillatory rheology, shown in Figure 5. Overall, there was a reduction in the storage and loss moduli of the gel with the addition of even low concentrations of cobalt. This indicates that the addition of cobalt ions reduces the stiffness of the hydrogel. This bulk property may be due to the areas of low-density collagen fibrils caused by cobalt collagen interactions as they are structurally weaker over the bulk gel structure.
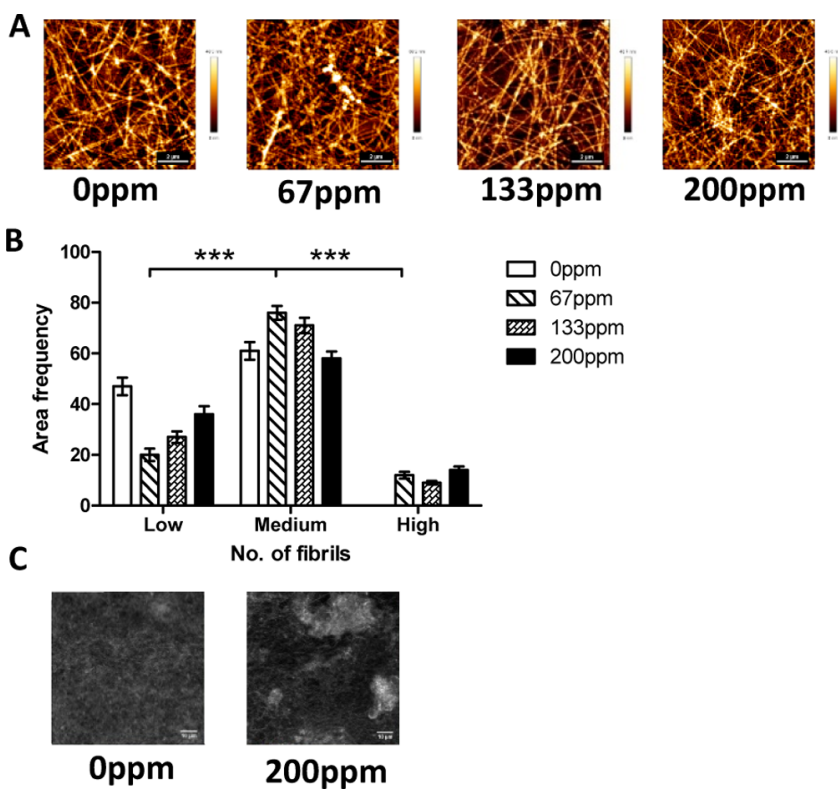

B

Figure 2. (A) AFM images of $1 \mathrm{mg} \mathrm{mL} \mathrm{m}^{-1}$ collagen with increasing levels of cobalt ions. (B) Density histogram indicating the number of fibrils present within a 5000 pixel $^{2}$ area. Fibrils were separated into three categories, low (4-6 fibrils), medium (7-10 fibrils), and high (11-13 fibrils), and the number of areas with distinct number of fibrils was tallied to obtain the area frequency. (C) Confocal reflectance images of $1 \mathrm{mg} \mathrm{mL}{ }^{-1}$ collagen with added cobalt ions.
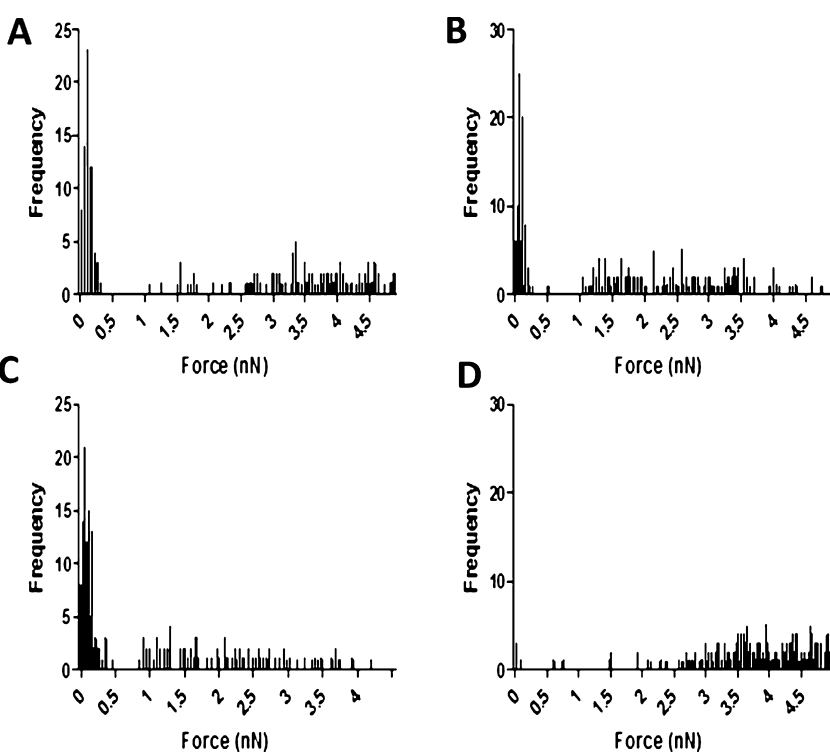

Figure 3. Force curves of CoCol gels at 0 (A), 67 (B), 133 (C), and $200 \mathrm{ppm}$ (D) indicating the adhesion force of collagen fibrils.

As the underlying structure of collagen effects the growth and proliferation of cells, various biological assays were performed. MC3T3 cells maintain a relatively high viability when seeded onto CoCol gels up to a cobalt concentration of $133 \mathrm{ppm}$, as shown by the confocal fluorescence images in Figure 6A. In comparison, MC3T3 cells seeded within a cobalt-doped culture medium only maintained a relatively high viability up to a cobalt concentration of $67 \mathrm{ppm}$, as shown in Figure $6 \mathrm{~B}$. This further indicates that any cell response is not due to the direct interaction between the cells and the cobalt ions. Furthermore, the presence of cobalt ions within the 

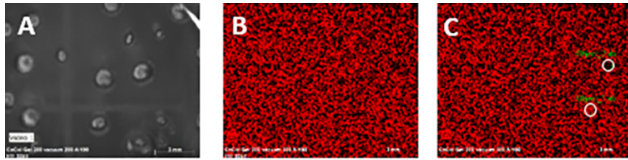

D

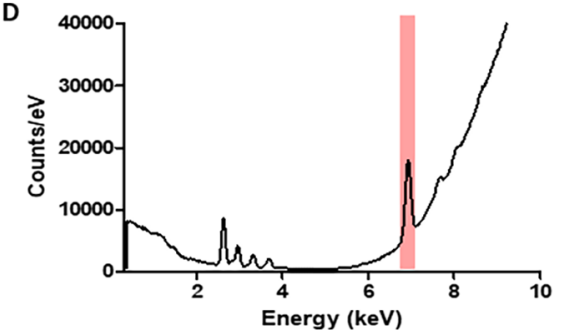

E

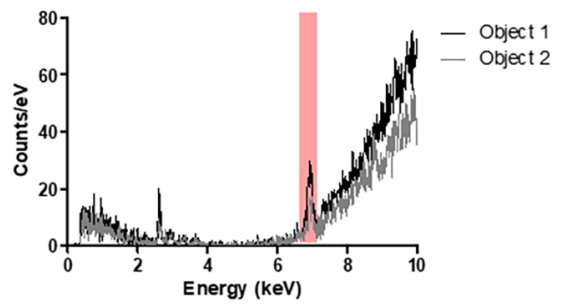

$\mathbf{F}$

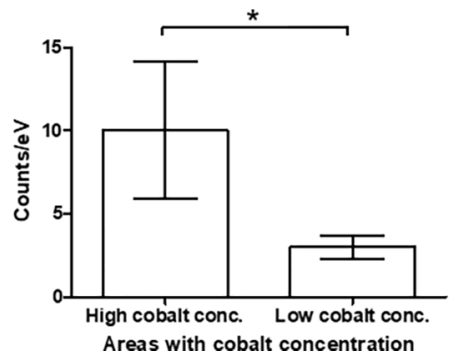

Figure 4. X-ray fluorescent measurements of cobalt distribution in a 200 ppm collagen hydrogel. (A) Bright-field image of bulk hydrogel, (B) relative cobalt intensity throughout hydrogel, (C) an example of positions of object 1 and object 2 in relation to bulk hydrogel, (D) spectra of gel with cobalt highlighted, (E) spectra of both object 1 and object 2 used to obtain an average of a 3 -fold increase in cobalt fluorescence, and (F) average cobalt concentration of all object areas separated by relatively high and low concentrations, giving a $P$ value $<0.1$.

collagen hydrogel caused a change in the morphology, with an initial increase in actin filament elongation prior to reduction in the cellular cytoskeleton entirely, as shown in Figure 6C. Metabolic activity of the cells is reduced with the addition of cobalt ions as shown by the 3-(4,5-dimethylthiazol-2-yl)-2,5diphenyltetrazolium bromide (MTT) assay. Figure 6D shows an $82 \%$ decrease in the absorbance showing a decrease in active mitochondria at a cobalt concentration of $100 \mathrm{ppm}$. The alamarBlue assay and trypan blue assay indicate that the proliferation of cells significantly decreased when low concentrations of cobalt ions were added to the collagen structure. This decrease was significant for both cells seeded onto CoCol gels and cells seeded within a cobalt-doped culture medium as shown in Figure 6E,F. This suggests that the structure of $\mathrm{CoCol}$ gels is directly affecting the way in which cells attach and therefore proliferate, causing a reduction in viability that correlates with the cobalt concentration.

To experimentally determine the preferential binding site between cobalt ions and collagen, Raman spectroscopy and differential scanning calorimetry (DSC) of both 0 and 200 ppm of cobalt-doped collagen samples were performed. Raman

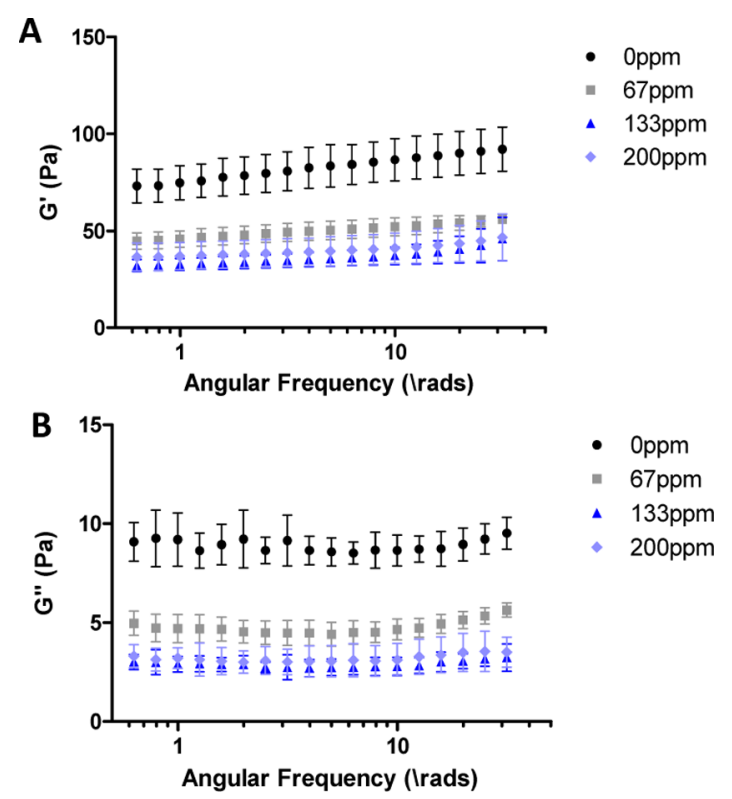

Figure 5. $G^{\prime}(\mathrm{A})$ and $G^{\prime \prime}$ (B) from a frequency sweep of collagen hydrogels with the addition of an increasing concentration of cobalt ions.

spectra of both 0 and $200 \mathrm{ppm}$ collagen samples showed characteristic protein peaks such as the $\mathrm{C}=\mathrm{O}$ peak at $1606.75 / 1607.76 \mathrm{~cm}^{-1}$ and the secondary amide peak at 1205.08/1207.25 $\mathrm{cm}^{-1}$, as shown in Figure 7A. ${ }^{47}$ However, in order to determine any subtle differences between each sample, principal component analysis (PCA) was performed on the data set after cosmic ray removal. The majority of information was described by the first two principal components. Figure $7 \mathrm{~B}$ corresponds to the scores plot $^{48}$ for all the Raman spectra, within which two clusters separating the 0 ppm sample and $200 \mathrm{ppm}$ sample were obtained. The position of relative differences between the 0 and $200 \mathrm{ppm}$ samples was determined through plotting the loading vectors as a function of wavenumber. ${ }^{49}$ The plot of the first loading, shown in Figure $7 \mathrm{C}$, indicates the most prominent changes between the spectra through intense peaks. ${ }^{48}$ It was found that the largest variation between the Raman spectra of the $0 \mathrm{ppm}$ sample and the $200 \mathrm{ppm}$ sample occurred at 869 and 1000 $\mathrm{cm}^{-1}$, respectively.

These wavenumbers correspond to a $\mathrm{C}-\mathrm{O}$ bond, ${ }^{47}$ with this vibration being suppressed within the $200 \mathrm{ppm}$ sample. DSC results show that bulk water within the $200 \mathrm{ppm}$ collagen sample is not bound as strongly as that within the $0 \mathrm{ppm}$ collagen sample. All the bound water within the $200 \mathrm{ppm}$ collagen sample is removed at a temperature of $120.3,5{ }^{\circ} \mathrm{C}$ lower than that of the $0 \mathrm{ppm}$ collagen sample. In addition, the energy required to completely remove the water bound within the $0 \mathrm{ppm}$ collagen sample is $14 \%$ more than that within the 200 ppm collagen, as shown in Figure 7D. This highly suggests that cobalt is binding to the collagen fibril, reducing the number of tightly bound water molecules surrounding the protein.

\section{DISCUSSION}

The introduction of cobalt ions to collagen hydrogels causes significant effects in both the gel structure and the way in which cells attach to the collagen. These ions cause a change in the kinetics of fibril formation. The elongation of the growth 


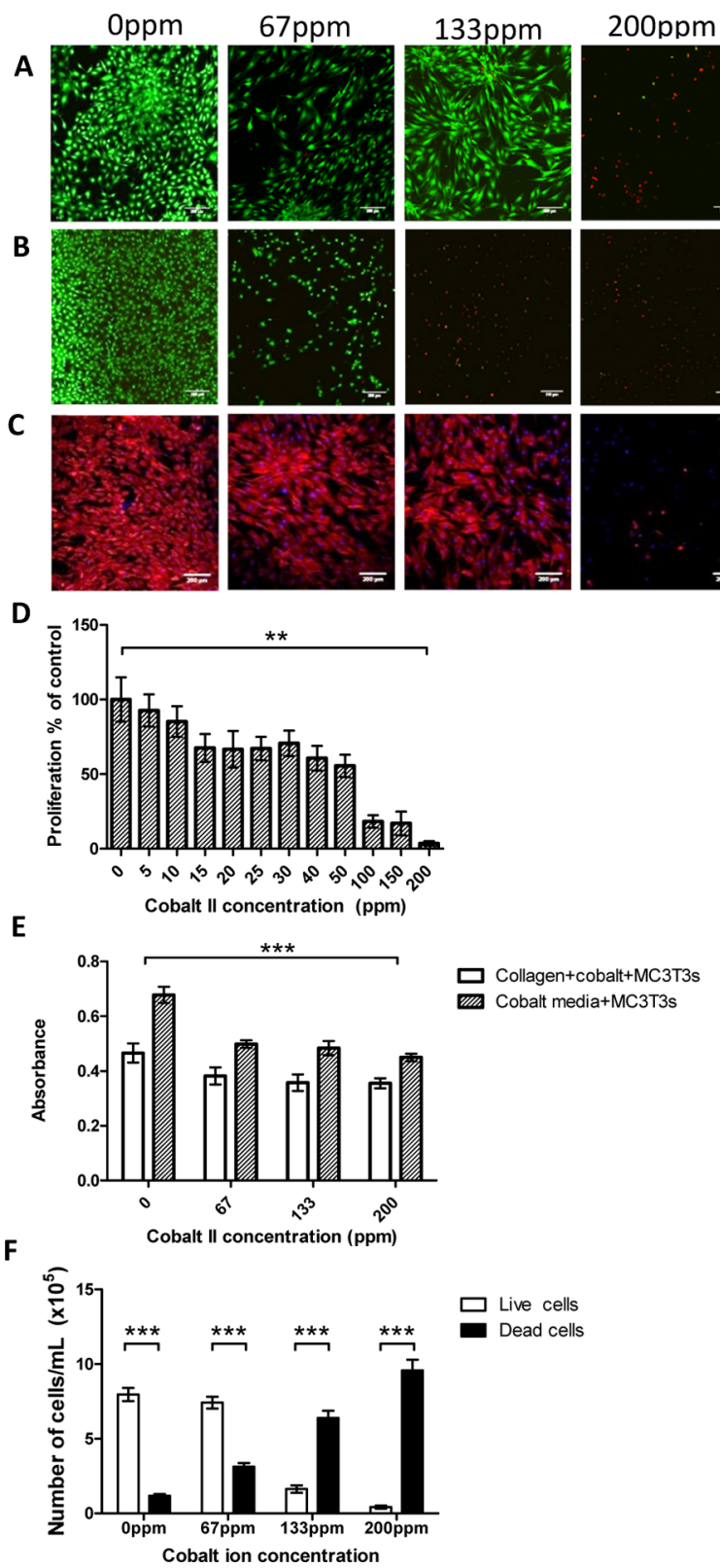

Figure 6. Live/dead images of MC3T3 cells stained with calcein AM and propidium iodide seeded onto both $\mathrm{CoCol}$ gels (A) and into cobalt-infused media (B). (C) Actin and 4',6-diamidino-2-phenylindole (DAPI) staining of MC3T3 cells cultured onto $\mathrm{CoCol}$ gels. (D) MTT assay of MC3T3 cells cultured in cobalt-infused media. (E) alamarBlue assay of MC3T3 cells cultured on CoCol gels and in cobalt-infused media. (F) Trypan blue assay quantifying the number of live and dead cells with increasing cobalt concentration.

phase indicates that cobalt ions directly interact with the collagen fibrils. On incubation, the CoCol gels released a maximum of only $8 \mathrm{ppm}$ of cobalt, suggesting that there is a strong interaction between the cobalt ions and the hydrogel, preventing the cobalt ions from diffusing out of the gel. AFM and confocal reflectance images show how increasing the concentration of cobalt increases areas of high-density collagen. A density histogram indicates that the addition of cobalt to the collagen matrix causes a high number of fibrils per unit area. These regions suggest that the cobalt ions ionically interact with the matrix to create areas of agglomeration, indicating that the cobalt ions are most likely present within

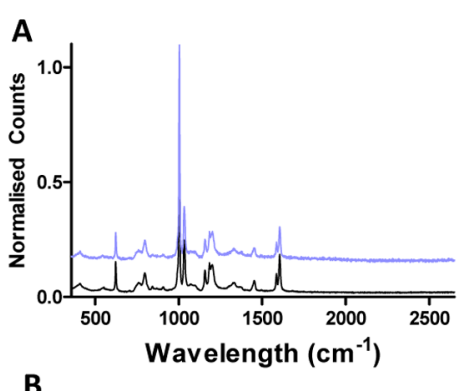

- Oppm Collagen Sample 200ppm Collagen Sample

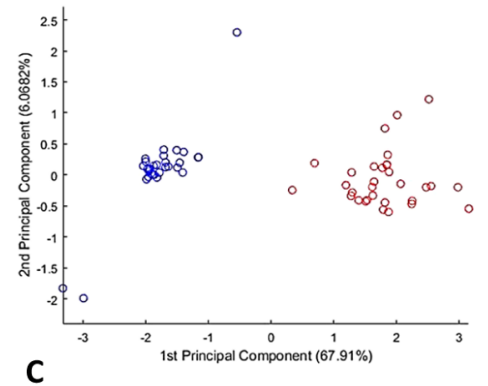

Oppm collagen sample 200ppm collagen sample
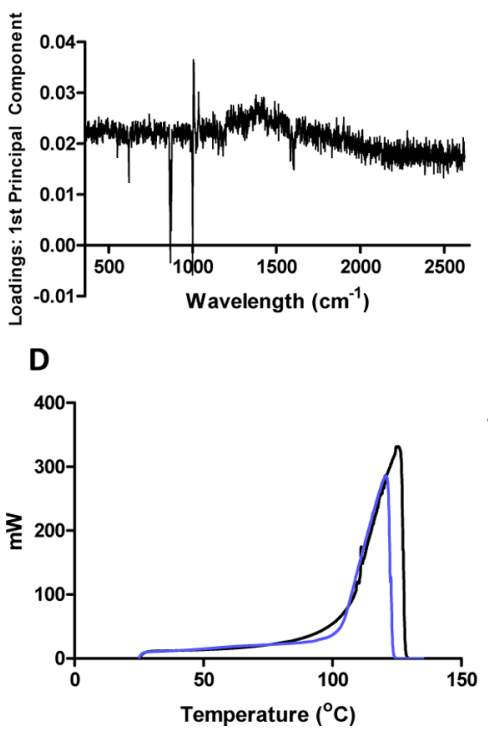

Figure 7. (A) Raman spectra of both 0 ppm collagen and 200 ppm collagen hydrogels, (B) score plots of the first two principal components, (C) loading plots of the first principal component indicating changes at 1000 and $869 \mathrm{~cm}^{-1}$, and (D) DSC measurements indicating a reduction in energy required to remove bulk water between 0 and $200 \mathrm{ppm} \mathrm{CoCol}$ gels.

these high-density regions. Overall, this causes an increase in the heterogeneity of the local collagen matrix. Force spectroscopy measurements indicated that a concentration of $200 \mathrm{ppm}$ produced softer fibrils than that of $0 \mathrm{ppm}$. Furthermore, samples containing 67 and 133 ppm had areas of both stiff and soft fibrils, whereas 200 ppm only had soft fibrils. This may be due to a decrease in cross-linking sites between fibrils, as they are blocked by cobalt ions.

Cobalt distribution throughout the bulk of the hydrogel, determined via XRF-, further indicates that the collagen fibril agglomeration sites are formed because of an increase in the localized cobalt concentration. As there was a 3 -fold relative increase in cobalt concentrations at select sites, it can be assumed that these areas correspond to high-density collagen areas. 
Regions of high and low densities of collagen make the overall bulk structure less stiff. Oscillatory rheology shows a decrease in the storage and loss moduli with even low concentrations of cobalt, indicating a reduction in overall stiffness. This may be due to the low-density areas of collagen which act as weak points within the structure. The increase in ionic bonding between collagen fibrils and cobalt ions prevents the interactions forming between collagen fibrils themselves and therefore reduces the overall stiffness of the hydrogel structure.

As we determined from Figure 1C, there was insignificant leaching of cobalt ions from the collagen hydrogel to have any effect on cell proliferation and viability. Therefore, any decrease is due to the change in the matrix formation alone. Fluorescence images suggest that the manipulation of collagen fibrils by the introduction of cobalt ions results in not only a decrease in cell viability but also a change in the morphology of the cells. This was further confirmed by phalloidin and DAPI staining, showing that at low concentrations of cobalt, there was an elongation of actin filaments, preventing the alignment of cells. Increasing the concentration of cobalt ions also caused a reduction in the actin filament production. This may be due to an increase in the heterogeneity of the bulk matrix. Cell viability staining indicated that there was no overall cell death until $200 \mathrm{ppm}$ of cobalt was added, however, because the heterogeneous distribution of cobalt in collagen cell viability varied between 67 and $133 \mathrm{ppm}$. Force measurements indicated that at $200 \mathrm{ppm}$, fibrils formed an overall soft material. This may explain why cell death significantly increased at $200 \mathrm{ppm}$ as the fibrils were not stiff enough to support cell growth. The MTT assay, alamarBlue assay, and trypan blue assay further suggest that the manipulation of collagen fibrils by cobalt ions prevents cells from behaving normally. The decrease in cell proliferation with an increase in cobalt ions may imply that poorly formed collagen fibrils, resulting in a heterogeneous matrix, cannot facilitate signaling between cells. The reduction in attachment sites between the cell and the collagen fibril results in a rounding-off of the cells, preventing the cells from signaling to each other and proliferating.

When cobalt(II) chloride is dissolved in water, the predominant cation is $\left[\mathrm{Co}\left(\mathrm{H}_{2} \mathrm{O}\right)_{6}\right]^{2+} \cdot{ }^{50}$ As $\left[\mathrm{Co}\left(\mathrm{H}_{2} \mathrm{O}\right)_{6}\right]^{2+}$ is a positive complex in solution, it will interact with negatively charged amino acid residues along the collagen chains. Negatively charged collagen chains can interact with water molecules and form a dense and strongly bound hydration monolayer. Four water molecules of the monolayer bind with the carbonyl and hydroxyl groups of both glycine and hydroxyproline in collagen, as shown in Figure $8 \mathrm{~A}^{51,52}$ Proline, however, is not associated with collagen hydration, participating only in interchain hydrogen bonding. ${ }^{53}$ Initial hydration of the collagen molecule, referred to as nonfreezing bound water, is formed when water binds to hydrophilic amino acid residues of the collagen. Once all hydrophilic sites are filled, the water binds to nonpolar regions of the collagen, referred to as freezing bound water, completing the hydration monolayer, Figure $8 \mathrm{~B}$. $^{52}$

As there is a lack of direct interactions between neighboring collagen molecules, it is assumed that hydrogen bonding of water bridges between residues in neighboring molecules contributes to the matrix assembly. Intrachain water bridges are dependent on the local environment and are typically two to three molecules long. Water bridges form in three ways,
A

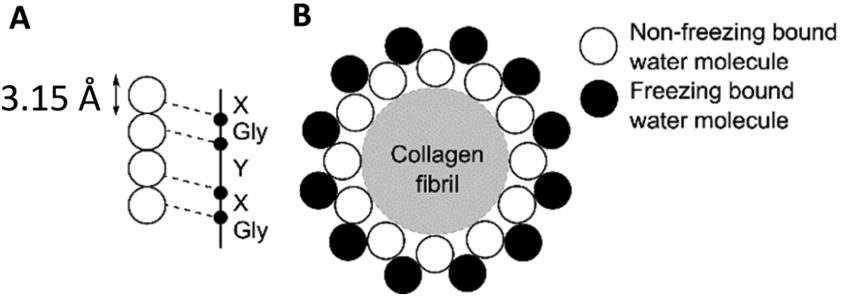

Figure 8. Schematic diagram depicting the cross section of the binding sites of water with a collagen fibril, not to scale. (A) Four water molecules per three collagen residues. (B) Nonfreezing and freezing bound water layers surrounding the collagen fibril. Image adapted from Fullerton and Amurao. ${ }^{49}$

either between two hydroxyl groups, $\mathrm{C}-\mathrm{O} \cdots$ water $_{n} \cdots \mathrm{O}-\mathrm{C}$; between two carboxyl groups, $\mathrm{C}=\mathrm{O} \cdots$ water $_{n} \cdots \mathrm{O}=\mathrm{C}$; or between a hydroxyl group and a carbonyl group in adjacent molecules, $\mathrm{C}-\mathrm{O} \cdots$ water $_{n} \cdots \mathrm{O}=\mathrm{C}^{47}$

When $\left[\mathrm{Co}\left(\mathrm{H}_{2} \mathrm{O}\right)_{6}\right]^{2+}$ is introduced into the collagen matrix, it may interact either directly with individual collagen fibrils or with water molecules of the nonfreezing bound water layer, depicted in Figure 9A,B. We then examined possible underlying mechanisms responsible for cobalt collagen

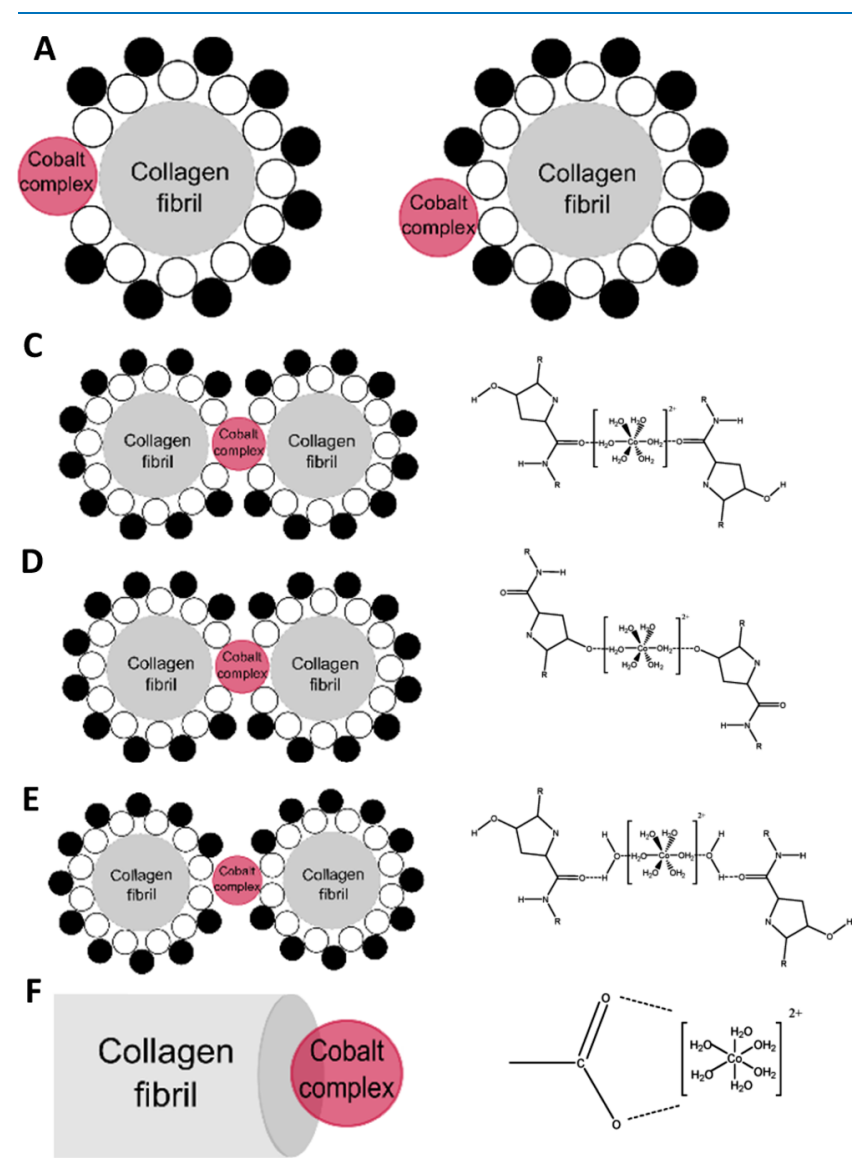

Figure 9. Schematic diagram depicting the binding sites of a cobalt complex with a collagen fibril, not to scale. (A) Cobalt complex interacting with the collagen fibril directly. (B) Cobalt complex interacting with the nonfreezing water layer surrounding the collagen fibril. Proposed binding sites of a cobalt complex with a collagen fibril where interactions occur with the carboxylic group (C), hydroxyl group (D), forming a water bridge (E), or interactions with the hydroxyl structure in the carboxylic terminal $(\mathrm{F})$. 
interaction. Direct interaction of cobalt complexes with collagen molecules occurs through either the carbonyl groups present in both glycine and hydroxyproline or the hydroxyl group present in hydroxyproline alone, shown in Figure 9C,D. If the cobalt complex interacts with the nonfreezing bound water layer, then hydrogen bonds will form between the water molecules and the cobalt complex, incorporating the complex into the water bridge, shown in Figure 9E. In addition, cobalt may also interact with the carboxylic terminal of the collagen molecule, as shown in Figure 9F.

Raman spectroscopy showed suppression of a secondary alcohol vibration, with a reduction in the $\mathrm{C}-\mathrm{O}$ bond peak, between 0 and $200 \mathrm{ppm}$ collagen samples. This suggests that cobalt ions interact specifically with a hydroxyl group. ${ }^{52-54}$ Although this is present within the hydroxyproline, previous studies into complex interactions with collagens show preferential binding to terminal sites of the molecule. ${ }^{55}$ Therefore, it is more likely that the cobalt ions interact with the hydroxyl structure at the carboxylic terminal. DSC measurements showed that a $14 \%$ decrease in energy is required to remove bound water from the $200 \mathrm{ppm} \mathrm{CoCol} \mathrm{gel.}$ This suggests that cobalt ion interactions prevent water binding either by blocking of the binding sites or altering the assembly of the collagen molecules, preventing these binding sites from being accessible.

\section{CONCLUSIONS}

We have shown that cobalt ions have an adverse effect on the way in which collagen fibrils form. An increase in the cobalt ion concentration lengthens the time it takes for a collagen matrix to form and produce areas of both high-density and lowdensity collagen fibrils. This causes an increase in the structural heterogeneity, reducing the overall stiffness of the collagen hydrogel. This change in matrix formation prevents osteoblasts from attaching and proliferating at a normal rate, changing the cell morphology and ultimately resulting in a decrease in cell viability. We have also suggested that the binding site for cobalt ions lies with the hydroxyl group present in the Cterminal. This leads toward a reduction in crucial stabilizing bonds within matrix formation, explaining the decrease in matrix stiffness and reduction in energy required for bulk water loss.

This gives further insights into the underlying mechanisms that result in unexpected failures of MOM implants. As we have shown, the collagen matrix is adversely affected, and it is likely that the extensive necrosis seen is more a result of the metal debris changing the ECM structure rather than interacting with the surrounding cells directly.

\section{MATERIALS AND METHODS}

Cobalt-doped collagen hydrogels (CoCol gels): Cultrex rat collagen type I (AMS Biotechnology Europe Marketplace, UK) supplied at a concentration of $5 \mathrm{mg} \mathrm{mL}^{-1}$ was diluted to $3 \mathrm{mg}$ $\mathrm{mL}^{-1}$ using a phosphate-buffered saline (PBS) stock $(\mathrm{NaCl}$ $0.137 \mathrm{M}, \mathrm{KCl} 0.003 \mathrm{M}, \mathrm{Na}_{2} \mathrm{HPO}_{4} 0.008 \mathrm{M}, \mathrm{KH}_{2} \mathrm{PO}_{4} 0.0015$ $\mathrm{M})$. This stock was created by dissolving two Oxoid PBS tablets (Thermo Fisher Scientific, UK) in $200 \mathrm{~mL}$ of distilled water. The cobalt(II) chloride stock was made to a concentration of $72.67 \mathrm{mg} \mathrm{mL}^{-1}$ with double-distilled water. Collagen was further diluted to the desired concentrations using Dulbecco's modified Eagle's medium (DMEM) (SigmaAldrich, UK) or cobalt-infused DMEM and neutralized with 1
M sodium hydroxide (Sigma-Aldrich, UK) prior to incubation for $3 \mathrm{~h}$ to ensure gelation.

Turbidity. A UV-vis spectrophotometer (Cecil Instruments, UK) was used to measure the turbidity of collagen dispersion during the process of fibril formation. Temperature was maintained at $37^{\circ} \mathrm{C}$, and samples were placed into a disposable $1 \mathrm{~cm}$ plastic cuvette, with absorbance measured at $620 \mathrm{~nm}$.

Leaching Assay. CoCol gels were prepared using Co concentrations of $0,67,133$ and $200 \mathrm{ppm}$ within a 12 -well plate. PBS $(2 \mathrm{~mL})$ (as prepared above) was added to the wells, removed at the fixed time points, and frozen. A 7500ce inductively coupled plasma optical emission spectrometer (Agilent Technologies, USA) was used to determine the concentration of leached cobalt ions from the frozen PBS samples. A two-way analysis of variance (ANOVA) was used to obtain the statistics.

Atomic Force Microscopy. CoCol gels were prepared as indicated above but with a buffer of PBS to reduce building up of salt during dehydration. Samples were deposited onto a pressure cleaned silicon wafer using an adjustable micropipette, resulting in a thin collagen layer on the solid substrate. The gel was dehydrated in an incubator over $24 \mathrm{~h}$ and subsequently washed three times with distilled water. A $10 \times 10 \mu \mathrm{m}^{2}$ area of each sample was imaged by a NanoWizard 4 NanoScience AFM (JPK Instruments, Germany), using a cantilever with a frequency of $20 \mathrm{kHz}$ and a spring constant of $0.9 \mathrm{~N} \mathrm{~m}^{-1}$ in the intermitted contact mode. Fibril density was determined by counting the number of fibrils present in a $5000 \times 5000$ pixel area. For each concentration, 108 areas were allocated over three samples to determine overall fibril density. Fibril density was classified as low (4-6 fibrils), medium (7-10 fibrils), and high (11-13 fibrils) and determined by separating the number of fibrils into three distinct groups. A two-way ANOVA was used to obtain the statistics.

Reflectance Microscopy. CoCol gels were prepared within a $35 \mathrm{~mm}$ imaging dish with an N\#1.5 coverslip base. The gels were imaged with a $60 \times$ water immersion lens with a $488 \mathrm{~nm}$ laser on an Olympus FX1000 microscope in the reflection mode. A three-dimensional stack of fixed volume was acquired via multiple images in the $z$-direction and compressed into a two-dimensional plane.

Force Spectroscopy. CoCol gel $(16 \mu \mathrm{L})$ was added to a silicon wafer. A Dimension 3100 AFM with a NanoScope IV controller (Veeco, Santa Barbara, USA) was used to perform nanomechanical adhesion measurements. Silicon nitride cantilevers (Budget Sensors, UK), with a nominal spring constant of $0.3 \mathrm{~N} \mathrm{~m}^{-1}$, were used to carry out these force measurements in the PBS solution. One hundred force curves were acquired over a $10 \times 10 \mu \mathrm{m}^{2}$ area from each location (three separate locations on each $\mathrm{CoCol}$ gel sample) for analysis. The control experiment, without the presence of a collagen film, was conducted on a titanium substrate. Overall adhesion forces between the AFM tip and the collagen film, which is reflected by the hysteresis between approaching and retraction curves, were analyzed using Carpick's toolbox, which is a suite of MATLAB scripts.

X-ray Fluorescence. A high-resolution measurement of a $4.9 \mathrm{~mL}$ sample of $200 \mathrm{ppm} \mathrm{CoCol}$ gel was analyzed using a TORNADO M4 XRF (Bruker, UK). A scan area of $100 \mathrm{~ms}$ pixel $^{-1}$ was used with a speed of $30 \mathrm{~m} \mathrm{~s}^{-1}$ for three cycles. Ten object areas were taken over the entirety of the scan area over both high- and low-intensity regions. The differences in the 
height of the peaks were measured, and the average difference is used to give an increase in the relative concentration of cobalt ions. Mann-Whitney test was performed to determine the statistics between average cobalt counts with respect to high- and low-density areas.

Rheology. CoCol gels were prepared in Petri dishes at a collagen concentration of $1 \mathrm{mg} \mathrm{mL}^{-1}$ and a gel height of $1 \mathrm{~mm}$. Samples were maintained at a temperature of $37^{\circ} \mathrm{C}$ for $5 \mathrm{~min}$ prior to measurement. Rheological measurements were taken with an AR-G2 rheometer (TA Instruments), using a parallel plate geometry with a gap of $1 \mathrm{~mm}$. A sandblasted plate was used to minimize the wall slip, and samples remained in the Petri dishes during measurement. Strain sweeps were taken, at a frequency of $1 \mathrm{~Hz}$, in order to establish the limit of the linear viscoelastic region, the lowest value of which was found to be 0.01 , for samples doped with $200 \mathrm{ppm}$ of cobalt. Frequency sweeps were therefore performed at a strain of 0.01 over a frequency range of $0.01-10 \mathrm{~Hz}$.

Cell Culture. Mouse MC3T3-E1 (passage 23, ATCC CRL2593) osteoblast precursor cells were cultured in a $\alpha$-minimum essential medium ( $\alpha$-MEM) (Sigma-Aldrich, UK) supplemented with $1 \%$ penicillin-streptomycin (Sigma-Aldrich, UK), $10 \%$ fetal bovine serum (Sigma-Aldrich, UK), and $4 \%$ L-glutamine (Sigma-Aldrich, UK). Cells were maintained at 37 ${ }^{\circ} \mathrm{C}$ and $5 \% \mathrm{CO}_{2}$ within a humidified incubator.

Confocal Fluorescent Imaging. CoCol gels, at a collagen concentration of $1 \mathrm{mg} \mathrm{mL}$, were gelled within a 12-well uncoated $10 \mathrm{~mm}$ glass diameter MatTek dish. For cell viability experiments, MC3T3 cell suspensions were seeded at a density of 200000 cells $\mathrm{mL}^{-1}$ onto the gel surface and grown within the supplemented $\alpha$-MEM (culture medium). In addition to this, MC3T3 cells were seeded at the same density onto the base of the wells. These were grown within the culture medium which had been doped with cobalt concentrations of 0,67 , 133, and $200 \mathrm{ppm}$. After culturing for 3 days, the medium was removed, and samples were washed with PBS. Calcein AM (Thermo Fisher Scientific, UK) and propidium iodide (Thermo Fisher Scientific, UK) dyes were prepared, with $300 \mu \mathrm{L}$ added to each well and incubated for $30 \mathrm{~min}$. For morphology experiments, MC3T3 cell suspensions were seeded at a density of 200000 cells $\mathrm{mL}^{-1}$ onto the surface of the CoCol gel and cultured for 3 days. The culture medium was then removed, and surfaces of the CoCol gel were washed twice with PBS (as prepared above). Cells were fixed within $4 \%$ formaldehyde for $15 \mathrm{~min}$ and washed as described before. Cells were then permeabilized with $0.5 \%$ Triton X-100 for 5 min. Staining with Alexa Fluor 488 Phalloidin and DAPI (Thermo Fisher Scientific, UK) allowed for visualization of the cytoskeleton and nuclei. Images were taken on a FV10-ASW confocal microscope with a $10 \times$ lens with image dimensions of $800 \times 800$ pixels.

MTT (3-(4,5-Dimethylthiazol-2-yl)-2,5-diphenyltetrazolium bromine) Assay. MC3T3 cells were seeded into a 48-well plate at a density of 7500 cells per well. Cells were initially cultured using supplemented $\alpha$-MEM for $24 \mathrm{~h}$. This was then aspirated and replaced with a cobalt-infused culture medium. Culture media containing Co(II) ions were prepared by adding the stock cobalt solution to the supplemented $\alpha$ MEM up to a concentration of $200 \mathrm{ppm}$. The $\mathrm{pH}$ was adjusted using $1 \mathrm{M}$ sodium hydroxide to neutral and diluted to the desired cobalt concentrations. Cells were cultured in cobalt media for a further $24 \mathrm{~h}$ prior to performing the MTT assay (Merck, USA). A stock solution of sterile-filtered $5 \mathrm{mg} \mathrm{mL}^{-1}$
MTT (Sigma, UK) in PBS was prepared and stored at $4{ }^{\circ} \mathrm{C}$ in light-deprived conditions. After culturing for 3 days, cobalt media was aspirated and replaced with $200 \mu \mathrm{L}$ of supplemented $\alpha$-MEM and $0.5 \mathrm{mg} \mathrm{mL}{ }^{-1}$ of MTT within each well. This was incubated for $2 \mathrm{~h}$ at $37^{\circ} \mathrm{C}$ to allow for the formation of formazan crystals. The MTT and $\alpha$-MEM solution was then removed, and $200 \mu \mathrm{L}$ of $0.22 \mu \mathrm{m}$ sterile filtered dimethyl sulfoxide was added to each well. The plate was then agitated for another $2 \mathrm{~h}$ at room temperature to dissolve formazan crystals. Absorbance was measured at 570 $\mathrm{nm}$ using a microplate reader (Promega, UK). A one-way ANOVA was conducted to determine the statistics.

alamarBlue Assay. MC3T3 cell suspensions were seeded onto the surface of the cobalt-doped collagen hydrogel at a concentration of 200000 cells $\mathrm{mL}^{-1}$ in 12 -well plates. After culturing for 3 days, the culture medium was aspirated, and $300 \mu \mathrm{L}$ of fresh medium mixed with $10 \%$ alamarBlue dye (Thermo Fisher Scientific, UK) was added. This was incubated at $37{ }^{\circ} \mathrm{C}$ for $4 \mathrm{~h}$ before $100 \mu \mathrm{L}$ of the medium was removed and placed into a 96-well plate. Absorption was read at 570 nm. A two-way ANOVA was conducted to determine the statistics.

Trypan Blue Assay. CoCol gels at concentrations of 0, 67, 133 , and $200 \mathrm{ppm}$ were gelled within a 12 -well plate. MC3T3 cell suspensions were seeded onto the gels at a concentration of 100000 cells $\mathrm{mL}^{-1}$. Samples were cultured for 3 days within a humidified incubator. In order to detach cells from the collagen hydrogel, $0.1 \%$ collagenase was added to each sample followed by incubation for an hour. To count cells, $100 \mathrm{~L}$ of media was added to $100 \mathrm{~L}$ of trypan blue, to produce a diluting factor of 2, and both live and dead cells were counted using a hemocytometer. A two-way ANOVA was conducted to determine the statistics.

Raman Spectroscopy. Two $1 \mathrm{~mL}$ samples of 0 and 200 ppm of cobalt-doped collagen gels were analyzed using the inVia Qontor Confocal Raman microscope (Renishaw, UK). PBS was used as a buffer within these gels to remove any additional signal from DMEM. A $60 \times$ water immersion lens was used to obtain Raman spectra. An extended scan over the spectral range of $100-4500 \mathrm{~cm}^{-1}$ was taken at random positions throughout each sample. Each scan had a $30 \mathrm{~s}$ acquisition using a $785 \mathrm{~nm}$ laser with the pinhole in at $10 \%$ power. Thirty scans of each sample were obtained, with cosmic ray removal performed on a wire. Averaging and normalizing of the data were obtained using Microsoft Office 365 Excel (2013). Reduction of the spectral range of $350-2650 \mathrm{~cm}^{-1}$ was performed using Prism 5 (GraphPad Software, 2007) to remove any background effects.

Principal Component Analysis. Raman spectra from both 0 and $200 \mathrm{ppm}$ CoCol samples were imported into MATLAB software (R2017a). The data set comprised 60 spectra in total, 30 from each sample. Before PCA, all spectra were normalized and reduced to a spectral range of 350-2650 $\mathrm{cm}^{-1}$ to remove any background interference. Once PCA was conducted over the data set, principal components were chosen based on the highest fraction of variance. Both the scores and loadings values were plotted with respect to wavelength to aid data interpretation.

Differential Scanning Calorimetry. Samples of 0 and $200 \mathrm{ppm}$ of cobalt-doped collagen gels were analyzed using a METTLER TOLEDO DSC 1 (METTLER TOLEDO, Schwerzenbach, Switzerland). Samples were weighed into 46 $\mu \mathrm{L}$ aluminum DSC pans (METTLER TOLEDO), capped with 
aluminum DSC lids (METTLER TOLEDO), and sealed with a press (METTLER TOLEDO). This was calibrated with indium and zinc standards. PBS was used as a buffer within the gels to remove any additional signal from DMEM. Experiments were conducted under a nitrogen flow rate of $50 \mathrm{~mL} \mathrm{~min}$, and samples were heated from 25 to $135{ }^{\circ} \mathrm{C}$ after gelation at a rate of $5{ }^{\circ} \mathrm{C} \mathrm{min}^{-1}$.

\section{ASSOCIATED CONTENT}

\section{S Supporting Information}

The Supporting Information is available free of charge on the ACS Publications website at DOI: 10.1021/acsomega.8b01048.

Circular dichroism data, further AFM data, and turbidity data relating to matrix formation with increasing cobalt ion concentration (PDF)

\section{AUTHOR INFORMATION}

\section{Corresponding Authors}

*E-mail: emm502@bham.ac.uk (E.M.M.).

*E-mail: L.M.Grover@bham.ac.uk (L.M.G.).

ORCID $\odot$

Emma M. McCarthy: 0000-0002-4318-2890

Zhenyu J. Zhang: 0000-0003-0243-2109

\section{Author Contributions}

All authors contributed to the design of the research. Experimental research was performed and analyzed predominately by E.M.M. and H.F. with force spectroscopy measurements completed by Z.J.Z. The manuscript was written by E.M.M. All authors reviewed and commented on the manuscript

\section{Notes}

The authors declare no competing financial interest.

\section{ACKNOWLEDGMENTS}

This work is supported in part by the EPSRC Sci-Phy-4-Health Centre for Doctoral Training (EP/L016346/1 to E.M.M.) and the NHIR Surgical Reconstruction and Microbiology Research (NIHR SRMRC to E.M.M.). All the data that were required to evaluate the conclusions made within this paper are present within the data presented and/or the supplementary data.

\section{REFERENCES}

(1) Nawabi, D. H.; Nassif, N. A.; Do, H. T.; Stoner, K.; Elpers, M.; Su, E. P.; Wright, T.; Potter, H. G.; Padgett, D. E. What causes unexplained pain in patients with metal-on metal hip devices? A retrieval, histologic, and imaging analysis. Clin. Orthop. Relat. Res. 2104, 472, 543-554.

(2) Mahendra, G.; Pandit, H.; Kliskey, K.; Murray, D.; Gill, H. S.; Athanasou, N. Necrotic and inflammatory changes in metal-on-metal resurfacing hip arthroplasties. Acta Orthop. 2009, 80, 653-659.

(3) Matthies, A. K.; Racasan, R.; Bills, P.; Blunt, L.; Cro, S.; Panagiotidou, A.; Blunn, G.; Skinner, J.; Hart, A. J. Material loss at the taper junction of retrieved large head metal-on-metal total hip replacements. J. Orthop. Res. 2013, 31, 1677-1685.

(4) Kwon, Y.-M.; Xia, Z.; Glyn-Jones, S.; Beard, D.; Gill, H. S.; Murray, D. W. Dose-dependent cytotoxicity of clinically relevant cobalt nanoparticles and ions on macrophages in vitro. Biomed. Mater. 2009, 4, 025018.

(5) Doorn, P. F.; Campbell, P. A.; Worrall, J.; Benya, P. D.; McKellop, H. A.; Amstutz, H. C. Metal wear particle characterization from metal on metal total hip replacements: Transmission electron microscopy study of periprosthetic tissues and isolated particles. J. Biomed. Mater. Res. 1998, 42, 103-111.

(6) Sieber, H.-P.; Rieker, C. B.; Küttig, P. Analysis of 118 secondgeneration metal-on-metal retrieved hip implants. J. Bone Jt. Surg. 1999, 81, 46-50.

(7) Koch, K. M.; Koff, M. F.; Bauer, T. W.; Shah, P. H.; Nencka, A. S.; Kaushik, S. S.; Potter, H. G. Off-resonance based assessment of metallic wear debris near total hip arthroplasty. Magn. Reson. Med. 2018, 79, 1628-1637.

(8) Lohmann, C. H.; Singh, G.; Willert, H.-G.; Buchhorn, G. H. Metallic debris from metal-on-metal total hip arthroplasty regulates periprosthetic tissues. World J. Orthoped. 2014, 5, 660-666.

(9) Langton, D. J.; Jameson, S. S.; Joyce, T. J.; Gandhi, J. N.; Sidaginamale, R.; Mereddy, P.; Lord, J.; Nargol, A. V. F. Accelerating failure rate of the ASR total hip replacement. J. Bone Joint Surg. Br. Vol. 2011, 93, 1011-1016.

(10) Davies, A. P.; Willert, H. G.; Campbell, P. A.; Learmonth, I. D.; Case, C. P. An Unusual Lymphocytic Perivascular Infiltration in Tissues around Contemporary Metal-on-Metal Joint Replacements. J. Bone Jt. Surg. 2005, 87, 18-27.

(11) Pearson, M. J.; Williams, R. L.; Floyd, H.; Bodansky, D.; Grover, L. M.; Davis, E. T.; Lord, J. M. The effects of cobaltchromium-molybdenum wear debris in vitro on serum cytokine profiles and $\mathrm{T}$ cell repertoire. Biomaterials 2015, 67, 232-239.

(12) Hart, A. J.; Matthies, A.; Henckel, J.; Ilo, K.; Skinner, J.; Noble, P. C. Understanding Why Metal-on-Metal Hip Arthroplasties Fail. J. Bone Jt. Surg., Am. Vol. 2012, 94, e22.

(13) Panichkul, P.; Fricka, K. B.; Hopper, R. H.; Engh, C. A. Greater Trochanteric Fragmentation after Failed Metal-on-Metal Hip Arthroplasty. Orthopedics 2015, 38, e447-e451.

(14) Sinnett-Jones, P. E.; Wharton, J. A.; Wood, R. J. K. Microabrasion-corrosion of a CoCrMo alloy in simulated artificial hip joint environments. Wear 2005, 259, 898-909.

(15) Jacobs, J. J.; Gilbert, J. L.; Urban, R. M. Corrosion of Metal Orthopaedic Implants*. J. Bone Jt. Surg., Am. Vol. 1998, 80, 268-282.

(16) Oldfield, J. W. Electrochemical theory of galvanic corrosion. Am. Soc. Test. Mater. 1998, 978, 5-22.

(17) Posada, O. M.; Tate, R. J.; Grant, M. H. Toxicity of cobaltchromium nanoparticles released from a resurfacing hip implant and cobalt ions on primary human lymphocytes in vitro. J. Appl. Toxicol. 2015, 35, 614-622.

(18) Posada, O.; Tate, R.; Meek, R. M.; Grant, M. In Vitro Analyses of the Toxicity, Immunological, and Gene Expression Effects of Cobalt-Chromium Alloy Wear Debris and Co Ions Derived from Metal-on-metal Hip Implants. Lubricants 2015, 3, 539-568.

(19) Haynes, D. R.; Rogers, S. D.; Hay, S.; Pearcy, M. J.; Howie, D. $\mathrm{W}$. The differences in toxicity and release of bone-resorbing mediators induced by titanium and cobalt-chromium-alloy wear particles. J. Bone Jt. Surg., Am. Vol. 1993, 75, 825-834.

(20) Hallab, N. J.; Jacobs, J. J. Chemokines associated with pathologic responses to orthopedic implant debris. Front. Endocrinol. 2017, 8, 5 .

(21) Ortega, R.; Bresson, C.; Fraysse, A.; Sandre, C.; Devès, G.; Gombert, C.; Tabarant, M.; Bleuet, P.; Seznec, H.; Simionovici, A.; Moretto, P.; Moulin, C. Cobalt distribution in keratinocyte cells indicates nuclear and perinuclear accumulation and interaction with magnesium and zinc homeostasis. Toxicol. Lett. 2009, 188, 26-32.

(22) Sargeant, A.; Goswami, T. Hip implants - Paper VI - Ion concentrations. Mater. Des. 2007, 28, 155-171.

(23) Jomova, K.; Valko, M. Advances in metal-induced oxidative stress and human disease. Toxicology 2011, 283, 65-87.

(24) Catelas, I.; Petit, A.; Vali, H.; Fragiskatos, C.; Meilleur, R.; Zukor, D. J.; Antoniou, J.; Huk, O. L. Quantitative analysis of macrophage apoptosis vs. necrosis induced by cobalt and chromium ions in vitro. Biomaterials 2005, 26, 2441-2453.

(25) Catelas, I.; Petit, A.; Zukor, D. J.; Antoniou, J.; Huk, O. L. TNF- $\alpha$ secretion and macrophage mortality induced by cobalt and chromium ions in vitro-Qualitative analysis of apoptosis. Biomaterials 2003, 24, 383-391. 
(26) Caicedo, M. S.; Pennekamp, P. H.; McAllister, K.; Jacobs, J. J.; Hallab, N. J. Soluble ions more than particulate cobalt-alloy implant debris induce monocyte costimulatory molecule expression and release of proinflammatory cytokines critical to metal-induced lymphocyte reactivity. J. Biomed. Mater. Res., Part A 2010, 93, 1312-1321.

(27) Samelko, L.; Caicedo, M. S.; Lim, S.-J.; Della-Valle, C.; Jacobs, J.; Hallab, N. J. Cobalt-Alloy Implant Debris Induce HIF- $1 \alpha$ Hypoxia Associated Responses: A Mechanism for Metal-Specific Orthopedic Implant Failure. PLoS One 2013, 8, e67127.

(28) Shah, K. M.; Wilkinson, J. M.; Gartland, A. Cobalt and chromium exposure affects osteoblast function and impairs the mineralization of prosthesis surfaces in vitro. J. Orthop. Res. 2015, 33, $1663-1670$

(29) Schrück, K.; Lutz, J.; Mändl, S.; Hacker, M. C.; Kamprad, M.; Schulz-Siegmund, M. Co(II)-mediated effects of plain and plasma immersion ion implanted cobalt-chromium alloys on the osteogenic differentiation of human mesenchymal stem cells. J. Orthop. Res. 2015, 33, 325-333.

(30) Anissian, L.; Stark, A.; Dahlstrand, H.; Granberg, B.; Good, V.; Bucht, E. Cobalt ions influence proliferation and function of human osteoblast-like cells. Acta Orthop. Scand. 2002, 3, 369-374.

(31) Pajarinen, J.; Lin, T.-h.; Nabeshima, A.; Jämsen, E.; Lu, L.; Nathan, K.; Yao, Z.; Goodman, S. B. Mesenchymal stem cells in the aseptic loosening of total joint replacements. J. Biomed. Mater. Res., Part A 2016, 105, 1195-1207.

(32) Hoppe, A.; Brandl, A.; Bleiziffer, O.; Arkudas, A.; Horch, R. E.; Jokic, B.; Janackovic, D.; Boccaccini, A. R. In vitro cell response to Cocontaining 1393 bioactive glass. Mater. Sci. Eng., C 2015, 57, 157163.

(33) Wu, C.; Zhou, Y.; Fan, W.; Han, P.; Chang, J.; Yuen, J.; Zhang, M.; Xiao, Y. Hypoxia-mimicking mesoporous bioactive glass scaffolds with controllable cobalt ion release for bone tissue engineering. Biomaterials 2012, 33, 2076-2085.

(34) Hoppe, A.; Jokic, B.; Janackovic, D.; Fey, T.; Greil, P.; Romeis, S.; Schmidt, J.; Peukert, W.; Lao, J.; Jallot, E.; Boccaccini, A. R. Cobalt-Releasing 1393 Bioactive Glass-Derived Scaffolds for Bone Tissue Engineering Applications. ACS Appl. Mater. Interfaces 2014, 6, 2865-2877.

(35) Hay, E. D. Cell Biology of Extracellular Matrix, 2nd ed.; Plenum Press: New York and London, 1991; pp 7-40.

(36) Hynes, R. O. The extracellular matrix: not just pretty fibrils. Science 2009, 326, 1216-1219.

(37) Frantz, C.; Stewart, K. M.; Weaver, V. M. The extracellular matrix at a glance. J. Cell Sci. 2010, 123, 4195-4200.

(38) Daley, W. P.; Yamada, K. M. ECM-modulated cellular dynamics as a driving force for tissue morphogenesis. Curr. Opin. Genet. Dev. 2013, 23, 408-414.

(39) Bonnans, C.; Chou, J.; Werb, Z. Remodelling the extracellular matrix in development and disease. Nat. Rev. Mol. Cell Biol. 2014, 15, $786-801$.

(40) Mayne, R.; Burgeson, R. E. Structure and Function of Collagen Types, 1st ed.; Academic Press: US, 1987; pp 1-37.

(41) Fratzl, P. Collagen: Structure and Mechanics; Springer: New York, 2008; pp 1-52.

(42) Kadler, K. E.; Baldock, C.; Bella, J.; Boot-Handford, R. P. Collagens at a glance. J. Cell Sci. 2007, 120, 1955-1958.

(43) Fang, M.; Goldstein, E. L.; Turner, A. S.; Les, C. M.; Orr, B. G.; Fisher, G. J.; Welch, K. B.; Rothman, E. D.; Holl, M. M. B. Type I Collagen D-Spacing in Fibril Bundles of Dermis, Tendon, and Bone: Bridging between Nano- and Micro-Level Tissue Hierarchy. ACS Nano 2012, 6, 9503-9514.

(44) Erickson, B.; Fang, M.; Wallace, J. M.; Orr, B. G.; Les, C. M.; Holl, M. M. B. Nanoscale structure of type I collagen fibrils: quantitative measurement of D-spacing. Biotechnol. J. 2012, 8, 117126.

(45) USHIKI, T. Collagen fibers, reticular fibers and elastic fibers. A comprehensive understanding from a morphological viewpoint. Arch. Histol. Cytol. 2002, 65, 109-126.
(46) Dobbs, H. S.; Minski, M. J. Metal ion release after total hip replacement. Biomaterials 1980, 1, 193-198.

(47) Socrates, G. Infrared and Raman Characteristic Group Frequencies Tables and Charts, 3rd ed.; John Wiley \& Sons LTD: Chichester, New York Weinheim, Toronto, Brisbane, Singapore, 2001; pp 11-339.

(48) Wold, S.; Esbensen, K.; Geladi, P. Principal Component Analysis. Chemom. Intell. Lab. Syst. 1987, 2, 37-52.

(49) González-Solís, J. L.; Martínez-Espinosa, J. C.; Salgado-Román, J. M.; Palomares-Anda, P. Monitoring of chemotherapy leukemia treatment using Raman spectroscopy and principal component analysis. Laser Med. Sci. 2014, 29, 1241-1249.

(50) Wertz, D. L.; Kruh, R. F. X-Ray Diffraction Study of Some Concentrated Cobalt(II) Chloride Solutions. J. Chem. Phys. 1969, 50, 4313-4317.

(51) Bella, J.; Brodsky, B.; Berman, H. M. Hydration structure of a collagen peptide. Structure 1995, 3, 893-906.

(52) Fullerton, G.; Amurao, M. Evidence that collagen and tendon have monolayer water coverage in the native state. Cell Biol. Int. 2006, $30,56-65$.

(53) Larkin, P. Infrared and Raman Spectroscopy: Principles and Spectral Interpretation, 2nd ed.; Elsevier: Netherlands, U.K., US, 2011; pp 85-113

(54) Frushour, B. G.; Koenig, J. L. Raman Scattering of Collagen, Gelatin, and Elastin. Biopolymers 1975, 14, 379-391.

(55) Birdi-Chouhan, G.; Shelton, R. M.; Bowen, J.; GoldbergOppenheimer, P.; Page, S. J.; Hanna, J. V.; Peacock, A.; Wright, A. J.; Grover, L. M. Soluble silicon patterns and templates: calcium phosphate nanocrystal deposition in collagen type 1. RSC Adv. 2016, 6, 99809-99815. 